\title{
Bedside Emergency Percutaneous Extracorporeal Membrane Oxygenator with Bicaval Dual-Lumen Cannula
}

\author{
Giuseppe Filiberto Serraino, MD, PhD, Federica Jiritano, MD, Michele Rossi, MD, \\ Pasquale Mastroroberto, MD \\ Cardiac Surgery Unit, University Magna Graecia of Catanzaro, V.le Europa, Germaneto, Catanzaro, Italy
}

\section{ABSTRACT}

Background: Veno-venous extracorporeal membrane oxygenation (VV-ECMO) support has emerged as a valuable rescue therapy in patients with severe acute lung failure. A new bicaval dual-lumen percutaneous cannula can be instituted with a single puncture of the right internal jugular vein under image guidance (fluoroscopy and/or trans-esophageal echocardiography) to support VV-ECMO. However, malpositioning of the dual-lumen catheter can jeopardize the efficacy of the ECMO therapy.

Case report: We report an emergency VV-ECMO instituted at a patient's bedside in the intensive care unit. Percutaneous insertion of a dual lumen-cannula was performed on a young patient that had undergone cardiac surgery. The patient had developed a fulminant post-operative pneumonia, leading to respiratory failure and septic shock. The procedure was done at night, without any image guidance other than a post-insertion chest $x$-ray. We compared the oxygen saturation of arterial blood gas taken from both the outflow and inflow ECMO ends, and the saturations were used as indirect proof of correct cannula placement. The VV-ECMO was successfully removed after nine days, as the patient was clinically and radiologically recovered.

Conclusion: This case reports a dual-lumen cannula insertion in an emergency setting, with minimal image support. It confirms the efficacy and the safety of VV-ECMO in the treatment of post-operative acute respiratory failure.

\section{INTRODUCTION}

Over the past two decades, veno-venous extracorporeal membrane oxygenation (VV-ECMO) support has emerged as a valuable rescue therapy that facilitates respiratory recovery in patients with severe lung failure. VV-ECMO is used mainly as a bridge until the lungs recover from the underlying disease, such as acute respiratory distress syndrome or pneumonia, or a bridge-to-transplant in end-stage lung diseases. There are two common configurations of VV-ECMO:

Received October 26, 2017; accepted April 27, 2018.

Correspondence: Federica Firitano, MD, Cardiac Surgery Unit - Dept. Experimental and Clinical Medicine, University of Magna Graecia, Catanzaro - 88100 - Italy; +39-0961-364-7058, fax: +39-0961-364-7142 (e-mail: fede.j@hotmail.it). single-site cannulation of the right internal jugular (RIJ) vein, and the more common two-site cannulation, involving the femoral and internal jugular veins.

Wang and colleagues described the double-lumen cannula (DLC) as being large enough to accommodate the high flows required for adult ECMO patients [Wang 2008]. One lumen drains deoxygenated blood from the superior and inferior venae cavae (SVC and IVC) through openings in the proximal and distal catheter, respectively, whereas the second lumen delivers oxygenated blood to the right atrium (RA). The advantages of a single site cannulation with a DLC mainly include minimizing blood recirculation between the ECMO's inflow and outflow tracts, sparing the patient's femoral vein, and allowing better mobilization possibilities (particularly the prone position) [Kuhl 2015]. However, the pitfall of using DLC is the need for correct positioning. In fact, a cannula's malposition could result in flow mixing between the outflow and inflow tracts, and can jeopardize the ECMO therapy. Fluoroscopy, together with trans-esophageal echocardiography (TEE), has been successfully employed to ensure the right positioning of the cannula [Javidfar 2011]. So far, a DLC has never been inserted without image guidance, and never at the patient's bedside.

Hereby, we report an emergency VV-ECMO with the Avalon Elite ${ }^{\circledR}$ DLC in a young, post-cardiac surgery patient who developed a fulminant post-operative pneumonia,

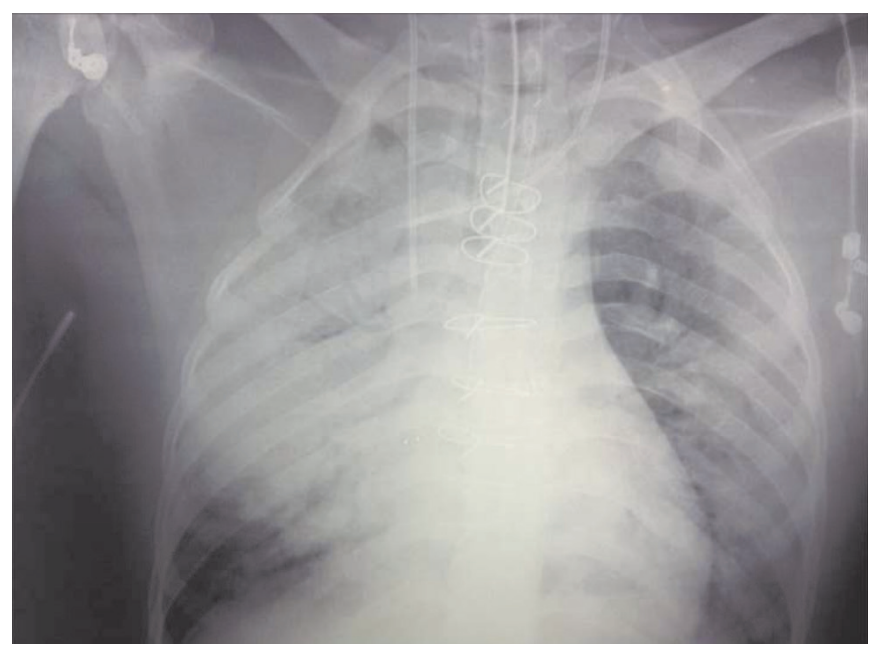

Figure 1. Chest $\mathrm{x}$-ray showing right lung consolidation. 


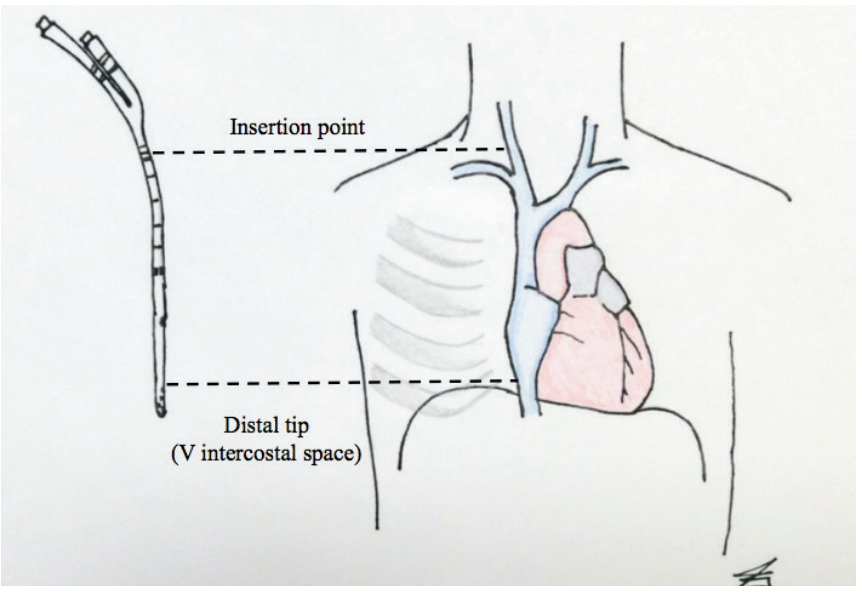

Figure 2. Measurement of the cannula's height, from the entry point at the neck to the fifth right intercostal space.

leading to acute respiratory failure and septic shock. The procedure was successfully done during the night, at the patient's bedside in the intensive care unit (ICU), without any image guidance other than a post-insertion chest $\mathrm{x}$-ray (CXR).

\section{CASE REPORT}

A 26-year-old man underwent aortic valve repair for severe aortic regurgitation. His initial recovery was uncomplicated: he was extubated on the day of surgery and transferred to the ward the following day. During the night on post-operative day (POD) three, he was transferred back to the ICU because of acute respiratory failure requiring urgent mechanical ventilation (MV). Despite very high ventilator settings (pressure control MV with $100 \%$ FiO2), severe hypoxia and hypercapnia persisted, leading to acidosis and circulatory failure. The CXR excluded tension pneumothorax, but showed an almost complete right lung consolidation (Figure 1). Trans-thoracic echocardiography showed good left ventricular function and a competent aortic valve. The patient became pyrexial at $39^{\circ} \mathrm{C}$ and developed a frank septic shock, requiring maximum adrenergic support with noradrenaline and adrenaline to maintain a mean arterial pressure around $50 \mathrm{mmHg}$.

After discussion, the intensivists and cardiac surgeons decided to implant a VV-ECMO to completely support the lungs. Our indication of $\mathrm{VV}$-ECMO was deteriorating hypoxia $\left(\mathrm{P}_{\mathrm{a}} \mathrm{O}_{2} / \mathrm{FiO}_{2}\right.$ ratio $\left.<70 \mathrm{mmHg}\right)$ under advanced $\mathrm{MV}$ $\left(\mathrm{FiO}_{2}>0.8\right.$ and peak inspiratory pressure $\left.>35 \mathrm{cmH}_{2} \mathrm{O}\right)$. He had a body mass index of $23.2 \mathrm{~kg} / \mathrm{m}^{2}$, so we used a $21 \mathrm{Fr}$. Avalon Elite® DLC. The cannula was inserted at the patient's bedside in ICU with a modified Seldinger technique, and serial RIJ vein dilatation up to the desired level as described by Javidfar [Javidfar 2011]. The procedure was done in emergency, at night, with no image support to check for correct cannula positioning. The cannula's approximate height was obtained by measuring, on the patient's body, the distance of an imaginary vertical line from the entry point at the neck to the fifth right intercostal space (Figure 2). These measurements were translated to the cannula, starting from its bottom.

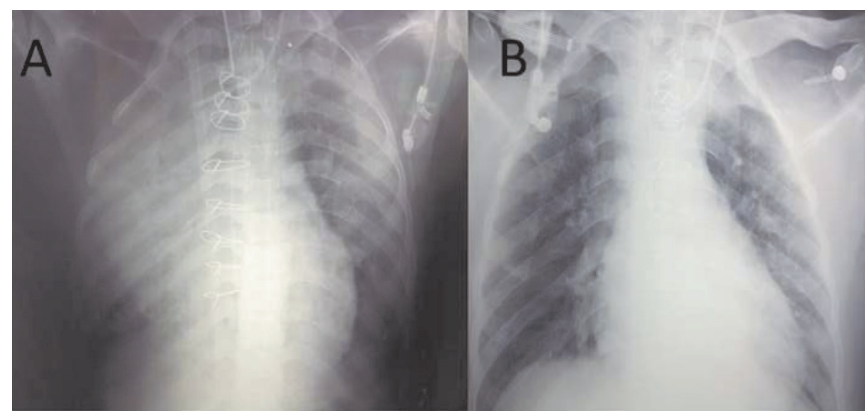

Figure 3. (A) Chest x-ray showing the VV-ECMO right inserted. (B) Resolution of the pneumonia on the chest $x$-ray.

During the insertion, the operator stood behind the patient's head. The cannula's outflow exit orifice was always kept at the operator's nine o'clock to favor its final positioning towards the tricuspid valve. The cannula's port was allowed to bleed back, and was clamped and carefully connected to the ECMO circuits (Quadrox-D oxygenator and Rotaflow Centrifugal Pump - Maquet, Wayne, NJ) to avoid any air entrapment. ECMO support was instituted at $1.5 \mathrm{~L} / \mathrm{min}$ after a post-insertion CXR confirmed the correct placement of the cannula (Figure $3 \mathrm{~A}$ ). The anticoagulation protocol consisted of a heparin drip that was titrated to keep an activated clotting time between 170 and 200 seconds. Packed red cells (PRCs) were transfused to keep hemoglobin levels above $9 \mathrm{~g} /$ dL. Ventilator settings were changed in order to have "lung rest" [Marhong 2014]. The hypoxia resolved, followed by a normalization of arterial blood gas (ABG) values and hemodynamic stability (Table).

Indirect proof of right cannula placement was obtained by an estimation of the rate of recirculation phenomenon using the following equation: (saturation of blood entering the membrane oxygenator - SvO2)/(SpostO2 saturation of blood leaving the membrane oxygenator - SvO2) x100. SvO2 was estimated by measuring venous saturation of blood from SVC via the central venous catheter. The calculated recirculation rate was less than 2\% [Van Heijst 2001].

Blood samples for microbiological cultures were collected and analyzed. Teicoplanin plus Ciprofloxacin were administered as initial empiric antimicrobial treatment. Blood cultures revealed a multi-drug resistant Staphylococcus aureus. The antibiotic therapy was then revised according to the microbiologic results, and Linezolid was administered. The patient was successfully weaned-off of VV-ECMO when the pneumonia was resolved, nine days after ECMO insertion (Figure 3B). Decannulation was done at the bedside, followed by manual compression for 30 minutes. Patient was extubated on POD 13 and discharged on POD 23, after an uneventful next post-operative period. At a one-year follow up, the aortic valve showed trivial regurgitation, and the CXR was normal.

\section{DISCUSSION}

When MV and other respiratory support techniques fail to maintain adequate oxygenation, VV-ECMO can be used 
Clinical Course for the Extracorporeal Membrane Oxygenation

\begin{tabular}{|c|c|c|c|c|c|c|c|c|c|c|}
\hline & One hour before ECMO & \multicolumn{7}{|c|}{ ECMO time (days) } & \multicolumn{2}{|c|}{ One hour after ECMO } \\
\hline Vital Signs & Heart Rate (bpm) & 145 & 120 & 105 & 107 & 98 & 92 & 89 & 90 & 86 \\
\hline \multirow{2}{*}{ Mechanical Ventilation } & $\mathrm{FiO}_{2}$ & 1.0 & 1.0 & 0.6 & 0.6 & 0.6 & 0.4 & 0.4 & 0.4 & 0.6 \\
\hline & $\begin{array}{l}\mathrm{PC} / \text { positive end-expirato- } \\
\text { ry pressure }\left(\mathrm{cmH}_{2} \mathrm{O}\right)\end{array}$ & $40 / 35$ & $12 / 10$ & $12 / 10$ & $12 / 10$ & $12 / 8$ & $10 / 8$ & $10 / 8$ & $10 / 8$ & $12 / 8$ \\
\hline \multirow[t]{4}{*}{ ECMO } & Sweep gas (L/min) & & 3 & 3 & 2.8 & 2.5 & 2.2 & 1.8 & 1.5 & \\
\hline & Pump flow $(\mathrm{l} / \mathrm{min})$ & & 3.2 & 3 & 3.4 & 3 & 2.8 & 2 & 1.4 & \\
\hline & $\begin{array}{l}\text { Unfractionated heparin } \\
\text { units } / \mathrm{h})\end{array}$ & 0 & 600 & 400 & 500 & 600 & 800 & 800 & 1000 & 0 \\
\hline & Activated clotting time $(\mathrm{s})$ & & 276 & 187 & 178 & 183 & 184 & 176 & 172 & \\
\hline \multirow[t]{3}{*}{ Arterial Blood Gas Analysis } & $\mathrm{pH}$ & 7.12 & 7.33 & 7.37 & 7.38 & 7.38 & 7.39 & 7.40 & 7.42 & 7.41 \\
\hline & $\mathrm{PaO}_{2}$ & 46 & 356 & 123 & 110 & 98 & 101 & 105 & 99 & 136 \\
\hline & $\mathrm{PCO}_{2}$ & 61 & 32 & 33 & 34 & 41 & 43 & 42 & 39 & 40 \\
\hline
\end{tabular}

$\mathrm{A} / \mathrm{C}$ indicates assist and control; ECMO, extracorporeal membrane oxygenation; $\mathrm{FiO}_{2}$, fraction of inspired oxygen; $\mathrm{PC}$, pressure control; $\mathrm{P} \mathrm{O}_{2}$, partial pressure of oxygen in arterial blood; $\mathrm{PCO}_{2}$, partial pressure of carbon dioxide.

effectively as a bridge to recovery, transplantation, or decision in select patients with severe respiratory failure. Historically, VV-ECMO was performed with two central cannulas, despite the advent of DLC, which makes single site cannulation an attractive option for VV-ECMO application [Banfi 2016]. Correct positioning of the Avalon cannula during placement of VV-ECMO is crucial to avoid complications and to ensure effective oxygenation. VV-ECMO using DLC from the RIJ vein access has significant advantages over conventional femoral-femoral and femoral-jugular VV-ECMO. Since DLC does not require a second femoral drainage cannula, the potential infection risk is reduced compared to the conventional VV-ECMO. Moreover, the patient is able to sit up, receive pulmonary toilet, and participate in limited physical therapy, all of which are important in critically ill patients.

In a retrospective analysis of their practice, Javidfar and colleagues reported good results with VV-ECMO in a series of 27 patients using the Avalon Elite ${ }^{\circledR}$ inserted with a modified Seldinger technique [Javidfar 2011]. The cannula was inserted in a hybrid operating room under fluoroscopy and/ or TEE guidance, ensuring correct cannula positioning. However, migration is a major complication of the Avalon cannula; a malpositioned DLC, in fact, significantly affects the ECMO support. In a series of 11 patients treated with VV-ECMO using the Avalon Elite ${ }^{\circledR}$, Bermudez reported two cases of cannula tip displacement in the RA [Bermudez 2010]. Tanaka and associates experienced two cases of migration of the DLC: one into the hepatic vein and the other into the right ventricle (RV) [Tanaka 2015]. The former was repositioned using echocardiographic guidance, without the use of a guidewire. The latter was repositioned using a guidewire from the femoral vein under fluoroscopy, without antegrade wire placement into the Avalon cannula, discontinuation of ECMO, or bleeding. To minimize cannula displacement, Bermudez suggested the insertion of the distal tip of the cannula well into the IVC [Bermudez 2010].

$<<$ NOTE TO AUTHORS: in the above paragraph, the numbering for the references is off. Bermudez was cited as 6 , when in the references section it is 7 , and Tanaka is cited as 7 , when in the reference section it is 8 . Please ensure that the references are correctly cited $>>$

When migration occurs, venous return from the distal end of the cannula may become compromised, which may result in decreased and unstable ECMO flow. The imbalance of the 
inflow from the tip of the cannula and the outflow from the middle portion of the cannula may result in inadequate oxygenation. Migration of the cannula may also cause injury to the surrounding tissue, including the RV, RA, and IVC [Hirose 2012]. Therefore, malposition of the cannula should be corrected as soon as possible. Decreased and/or unsteady ECMO flow and unexplained hypoxia are possible signs of migration. A CXR is diagnostic for this condition [Tanaka 2015].

What is remarkable in our case is that a DLC was implanted in an emergency situation with a percutaneous insertion done at the ICU bedside, without image guidance. The risk of an insertion-related injury or cannula malpositioning were weighted against the urgent need of ECMO therapy and the patient's rapid deterioration. Because of the effectiveness of the ECMO therapy established soon after, we did perform a TEE to check the cannula position once the patient was hemodynamically stable the following morning, which confirmed the correct positioning of the cannula. Another sign we judged to be an emergency index of proper cannula position was a low hemolysis recorded during the first five hours after ECMO implantation, supported by blood tests and low transfusion requirement (just 1 unit of PRCs during the ECMO period). We did not have any adverse events related to the cannula, (during the insertion, the ECMO therapy and the decannulation) such as bleeding, thrombosis, or infection at the cannulation site.

The simpler the use of a device is, the wider its application will be. We believe the advent of DLC was a breakthrough in the application of VV-ECMO. Even though we are far from recommending the abandonment of image support during the insertion of the Avalon Elite ${ }^{\circledR}$ DLC, we are pleased to report the transition of its insertion from the operation room to an intensive care environment.

\section{REFERENCES}

Banfi C, Pozzi M, Siegenthaler N, et al. 2016. Veno-venous extracorporeal membrane oxygenation: cannulation techniques. J Thorac Dis 8:3762-73

Bermudez CA, Rocha RV, Sappington PL, et al. 2010. Initial experience with single cannulation for venovenous extracorporeal oxygenation in adults. Ann Thorac Surg 90:991-5.

Hirose H, Yamane K, Marhefka G, et al. 2012. Right ventricular rupture and tamponade caused by malposition of the Avalon cannula for venovenous extracorporeal membrane oxygenation. J Cardiothorac Surg 7:36.

Javidfar J, Wang D, Zwischenberger JB, et al. 2011. Insertion of bicaval dual lumen extracorporeal membrane oxygenation catheter with image guidance. ASAIO J 57:203-5.

Kuhl T, Michels G, Pfister R, et al. 2015. Comparison of the avalon dual-lumen cannula with conventional cannulation technique for venovenous extracorporeal membrane oxygenation. Thorac Cardiovasc Surg 63:653-62.

Marhong JD, Telesnicki T, Munshi L, et al. 2014. Mechanical ventilation during extracorporeal membrane oxygenation. An international survey. Ann Am Thorac Soc 11:956-61.

Tanaka D, Pitcher HT, Cavarocchi N, et al. 2015. Migrated avalon venovenous extracorporeal membrane oxygenation cannula: how to adjust without interruption of flow. J Card Surg 30:865-8.

Van Heijst AF, van der Staak FH, de Haan AF, et al. 2001. Recirculation in double lumen catheter veno-venous extracorporeal membrane oxygenation measured by an ultrasound dilution technique. ASAIO J 47:372-6.

Wang D, Zhou X, Liu X, et al. 2008. Wang-Zwische double lumen cannula-toward a percutaneous and ambulatory paracorporeal artificial lung. ASAIO J 54:606-11. 